\title{
REVUES CRITIQUES
}

\section{LES TRÉMATODES DIGENEA DES SÉLACIENS}

\section{(PLAGIOSTOMES)}

\section{CATALOGUe PAR HÔTES. DISTRIBUTION GÉOGRAPHIQUe}

\author{
Par . Robert-Ph. DOLLFUS
}

Dans le présent catalogue, j'ai noté toutes les récoltes dont j'ai eu connaissance de distomes chez des sélaciens. J'ai indiqué, pour chaque observation, la référence bibliographique des ouvrages auxquels il faudra se reporter pour les descriptions et l'iconographie.

L'ordre suivi pour l'énumération des hôtes est, à très peu près, celui de l'ouvrage classique de Samuel Garman. (The Plagiostoma. Mem. Mus. Comparat. Zool. Harvard College, XXXVI, 1913).

On trouvera, avant le répertoire bibliographique, une liste des distomes avec, pour chaque espèce, un renvoi aux pages où il en est question.

\section{A. - SQUALOIDEI}

Famille des CHLAMYDOSELACHIDE

\section{Chlamyoselachus angeineus Garman 1884}

Otodistoma cestoides. (P. J. Van Beneden 1870). - C'est à ce distome, selon T. Odhner (1911, p. 517) que l'on doit rapporter le parasite signalé comme Distoma veliporum Creplin ou espèce voisine, chez cet hôte du Varangerfjord (Norvège), par Einar Lönnberg (1898, separ. p. 4).

Annales de Parasitologie, $\mathrm{T}$. XV, $\mathrm{N}^{\circ} 1 .-1^{\mathrm{er}}$ janvier 1937, p. 57-73. 


\section{Famille des HEXANCHIDE}

2. Hexanchus griseus (Bonnaterre 1788), Rafinesque $\mathbf{1 8 1 0}$

Syn. Notidanus griseus (Bonnaterre 1788), G. Cuvier 1817. Otodistoma veliporum. (Fr. Chr. Creplin 1837), J. Stafford 1904. - Ce distome a été signalé, chez cet hôte, sous les noms de :

a) Fasciola squali grisei Risso, par Diesing (1850, p. 347) ex-Risso (1810, p. 38), à Nice ;

b) Distoma veliporum Creplin, par : Creplin (1837, p. 310, 318 ; 1842, p. 336-339, pl. IX, fig. 1-2) en Sicile ; Diesing (1850, p. 367) en Sicile ; C. Parona (1887, p. 490, 497 ; 1896, p. 3), à Gênes ; M. Stossich $(1888$, p. 99) à Gênes ; Fr. Zschokke $(1887$, p. 266) à Naples ; M. Stossich $(1890$, p. 51) et E. Ninni (1900, p. 57) à Venise ; M. Stossich (1900, p. 97, pl. VI, fig. 1, œuf) à Trieste ; Fr. Sav. Monticelli (1893, p. 191) à Naples ;

c) Otodistoma veliporum (Crepl.), par T. Odhner (1911, p. 516) de la Méditerranée ou de l'Atlantique sud-européen; Georg Mühlschlag (1914, p. 199) origine inconnue (Musée de Berlin); L. Cuénot (1927, p. 247) à Arcachon (Gironde) ; Robert Ph. Dollfus (Mss.), de la côte française de l'Atlantique, par $47^{\circ} 20$ Lat. N., env. $175 \mathrm{~m}$. de profondeur (Pierre Desbrosses, leg. 19-4-1934).

\section{Heptanchus cinereus (Gmelin 1789), Müller et Henle 1841}

Syn. Heptranchias perlo (Bonnaterre 1788); Squalus cinereus (Gmelin 1789) ; Notidanus cinereus (Gmel. 1789), G. Cuvier 1829.

Otodistoma veliporum (Fr. Chr. Creplin 1837) J. Stafford 1904. Ce distome a été signalé, chez cet hôte, sous les noms suivants :

a) Distoma veliporum Creplin, par Fr. Zschokke (1887, p. 266) à Naples ; par Fr. Sav. Monticelli (1893, p. 191) à Naples.

b) Otodistoma veliporum (Creplin), par T. Odhner (1911, p. 516), de la Méditerranée ou de l'Atlantique sud-européen.

Famille des RHINCODONTIDAE

\section{Rhincodon typus A. Smith 1829}

Syn. Rhinodon typicus Müller et Henle 1841 ; Micristodus punctatus Gill 1865 ; Rhinodon pentelineatus Kishinouye 1901.

A. E. Shipley et J. Hornell (1905, p. 54) ont rapporté que de nombreux spécimens de "Distomum richiardii Lopez » avaient été récoltés dans la cavité péri-viscérale de ce Rhinodon des « Pearl Beds », de Ceylan. Il est cependant incertain que l'hôte 
était bien un Rhinodon, car, d'après Shipley et Hornell (1905, p. 53), il s'agissait du « basking shark » et si ce nom concerne, en principe, "Rhinodon typus », dans l'Océan Indien, il est aussi employé pour d'autres squales ; d'après Herdman, il s'agirait d'un Carcharias, mais un dessin d'un de ces "basking shark » des bancs perliers, soumis à Boulenger, a été identifié à Stegostoma tigrinum (Forster). C'est donc sans certitude que l'on compte Rhincodon typus A. Smith au nombre des hôtes de Probolitrema richiardii (C. Lopez).

\section{Famille des ORECTOLOBIDAE}

5. Ginglymostoma cirratum (Bonnaterre 1788)

Müller et Henle 1841

Syn. Nebrodes concolor (Rüppell 1837) ; Ginglymostoma concolor (Rüppell 1837), T. Cantor 1849.

A) T. Southwell $(1913$, p. 101) a signalé qu'il avait trouvé en abondance Anaporrhutum largum Lühe dans le cœlome de cet hôte, à Ceylan.

B) H.W. Manter $(1930$, p. 340$)$ a trouvé Distoma fenestratum Linton 1907, immature, dans le cœur, le foie, la rate, le rein, l'estomac d'un Ginglymostoma des Tortugas. L'exemplaire hébergeant ces parasites, dit Manter (1931, p. 385) était resté en aquarium pendant quelques semaines, avant d'être examiné. Aux Tortugas, D. fenestratum Linton a été trouvé par H. W. Manter dans 22 espèces de poissons, mais jamais à maiurité. L'adulte est inconnu.

6. Stegostoma varium (Seba 1761)

$=$ Stegostoma tigrinum (Forster 1781), Günther 1870

T. Southwell (1913, p. 101) a rapporté à Anaporrhutum largum Lühe des spécimens trouvés par Jenkins dans le coelome de cet hôte, de la côte d'Orissa. Ces spécimens, à contour plus circulaire, à sinuosités de la paroi interne de l'intestin pas bien marquées, à testicules et vitellogènes faiblement développés, ont été considérés par Southwell comme appartenant néanmoins à l'espèce de Lühe.

\section{Chiloscyllium indicum (Gmelin 1789)}

A) T. Southwell (1913, p. 101-102) a signalé la récolte d'un grand nombre d'Anaporrhutum albidum Ofenheim à la surface du foie de cet hôte, pris sur les Ceylon Pearl Banks. Ces spécimens 
différaient de la description d'Ofenheim en ayant la ventouse ventrale beaucoup plus grande et les testicules moins dispersés. Ce dernier fait, dit Southwell, peut être dû à ce que les testicules n'étaient pas complètement développés chez ces spécimens.

B) T. Southwell (1913, p. 101) a mentionné qu'il avait trouvé en grand nombre Anaporrhutum largum Lühe dans le cœlome de cet hòte, à Ceylan.

Famille des CATULIDE

8. Catulus caniculus (Linné 1758)

Syn. Squalus canicula Linné 1758 ; S. catulus Linné 1758 ; Scylliorhinus caniculus (L. 1758) Blainville 1816; Scyllium canicula (L. 1758), G. Cuvier 1817.

A) Ptychogonimus megastoma (Rud.) a été signalé chez cette roussette par: Jean Kuhn (1829, p. 463-465, pl. I, fig. 4-5), dans l'estomac, sous le nom de Distomum megastomum; côtes de France ;

K. Wedl (1855, p. 383, 394, pl. II $a$, fig. 16), dans l'estomac sous le nom de Dist. megastoma, à Trieste.

B) P. J. Van Beneden (1870, p. 3, 93, pl. IV, fig. 9') a désigné sous le nom de Distoma lutea Van Beneden, un distome trouvé dans l'intestin d'une roussette de la còte de Belgique. Ce parasite porte maintenant le nom de Diphtherostoma betencourti Monticelli 1893 (vide infra, p. 61), il n'est pas rare chez les roussettes de la côte française de l'Atlantique ; je l'ai retrouvé dans le rectum d'un caniculus à Roscoff (Finistère) (18-9-1913, un spécimen, rouge sur le vivant) et chez un caniculus venant de Saint-Servan (Ille-etVilaine) (15-8-1931, nombreux spécimens macérés).

C) G. Mühlschlag $(1914$, p. 200) a rapporté à Otodistoma veliporum (Creplin) des immatures (métacercaires ?) trouvés dans des kystes de la paroi de l'estomac de cette roussette (Musée de Berlin).

9 a. Catulus stellaris (Belon 1553), Garman 1913

Syn. Scyllium catulus G. Cuvier 1817 ; S. stellare (L. 1758) Fleming 1828.

A) Ptychogonimus megastoma (Rud.) a été signalé chez cette roussette par :

M. Stossich (1883, p. 118-119) sous le nom de Distomum soccus Molin, dans l'estomac, à Trieste et M. Stossich $(1898$, p. 38, 157) sous le nom de Distomum megastoma Rud. (= soccus Molin), dans l'estomac, à Trieste. 
F. Basile (1909) (fide C. Parona 1912, p. 38, 39, 361) sous le nom de Dist. soccus Molin ou Dist. megastoma, à Messine.

B) Diphtherostoma betencourti Monticelli. - Au Portel, près Boulogne-sur-Mer (Pas-de-Calais), dans l'estomac de cette roussette, Bétencourt trouva des spécimens de Distoma luteum Van Beneden ; ceux-ci furent étudiés par F. S. Monticelli (1890, p. 424426, 443-444, pl. XXII, fig. 21-27) d'abord sous le nom de Dist. luteum Van Ben., puis sous le nom nouveau de Distomum betencourti Monticelli (1893, p. 33, 34, 35, 43, 52, 84, 85, 88, 91, 95, 96, 102, 106, 107, 114, 146, 171, 186, 190-191, 216, 227, pl. VIII, fig. 121), en raison de l'existence d'un Distomum luteum K. E. von Baer 1826.

Stossich (1904, p. 193-197) fit entrer Dist. betencourti Monticelli dans son genre Diphtherostoma. Des spécimens types, eommuniqués par Monticelli, furent redécrits par Odhner (1911, p. 242-243), qui établit alors que ce genre devait être admis dans la famille des Zoogonidae.

\section{9 b. Catulus (= Scyllium) sp. plur.}

A) Ptychogonimus megastoma (Rud.), d'un Scyllium sp. des Hauts de Bréat, a été utilisé par Marcel Prenant (1922, p. 6, 32, 53, 172, pl. IV, fig. 4), pour ses recherches sur la cytologie du parenchyme (1).

B) Ernst von Ofenheim (1900, p. 169-180, 185-186, pl. III, fig. 9-12) a décrit, sous le nom d'Anaporrhutum richiardii Lopez, neuf spécimens d'un distome trouvé dans le colome d'un Scyllium sp. capturé dans les parages du Cap de Bonne-Espérance. (Collection du Musée de Berlin).

Ofenheim estima que ce distome était à la fois identique à celui de Lopez et congénérique d'Anaporrhutum albidum Ofenh.

A. Looss (1911, p. 204) a choisi albidum comme type du genre d'Ofenheim, qu'il réunit à Plesiochorus Looss dans la s. fam. des Anaporrhutinæ Looss (1901, p. 205). Peu après Looss (1902, p. 852-855) discuta longuement l'attribution du distome du Scyllium du Cap à l'espèce méditerranéenne de C. Lopez; il en conclut qu'il ne s'agissait pas de la même espèce, mais seulement du même genre et que ce genre n'était pas Anaporrhutum, d'où la

(1) Je m'abstiens, dans la présente publication, d'insister sur les références concernant Pt. megastoma (Rud.), où il n'est pas cité l'hôte d'origine. Par exemple : Monticelli $(1892 a, 1892 h, 1893)$, L. A. Jägerskiöld (1900), T. Odhner (1928), etc.... etc... n'ont pas indiqué de quels hôtes provenaient les spécimens qu'ils étudièrent. 
création du genre Probolitrema A. Looss (1902, p. 855, 857 , 858, 859, 860,863 définition et fig. B. VI schéma du genre), caractérisé par les testicules et les vitellogènes entièrement extracæcaux, avec pour type Pr. richiardii (Lopez) et pour seconde espèce «Pr. capense Looss (= Anaporrhutum richiardii Ofenheim nec Lo-

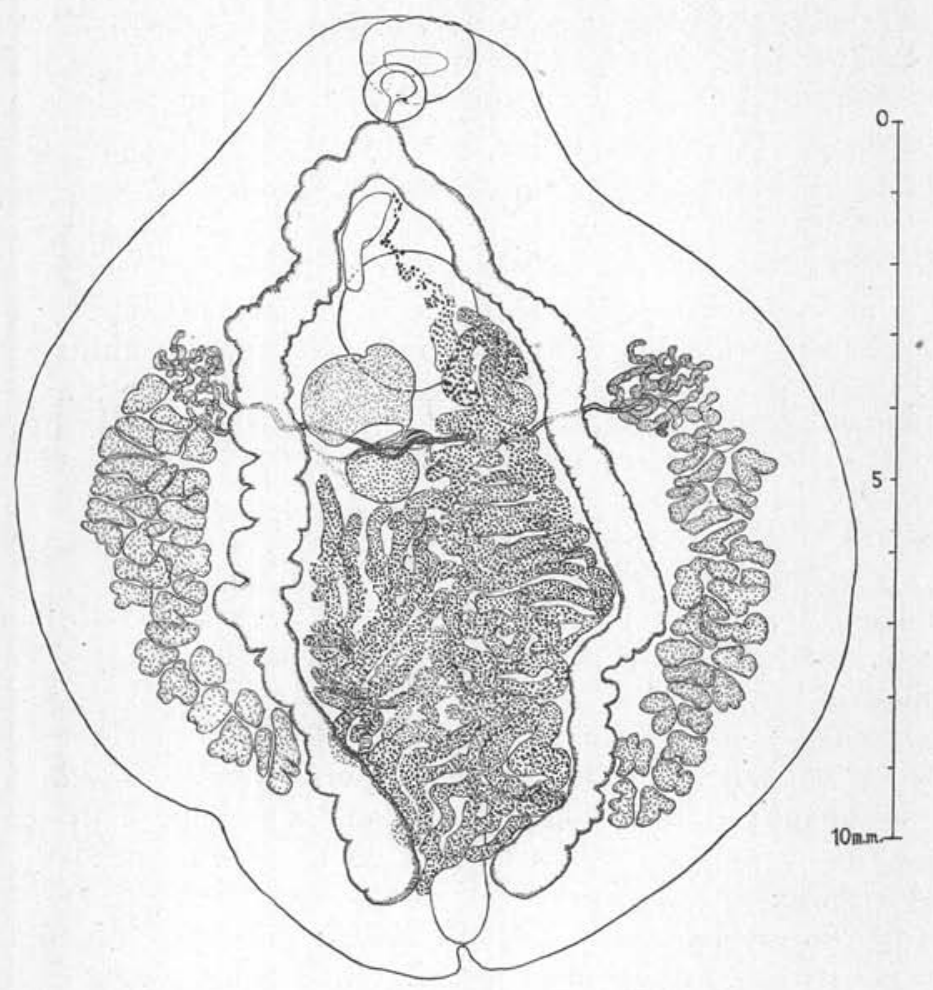

Fig. 1. - Probolitrema richiardii (C. Lopez 1888), individu vu par la face dorsale, préparation in toto.

pez) ». Selon Looss, capense se distinguerait par ses ventouses à peu près d'égal diamètre, de richiardii à ventouse ventrale plus grande que l'orale.

T. Odhner $(1910$, p. 14) a réexaminé les spécimens types de Probolitrema capense Looss, du Musée de Berlin, il en a donné les dimensions en précisant le diamètre des ventouses (pour les grands spécimens longs de $20 \mathrm{~mm}$., rapport des ventouses comme $3: 4$; pour les petits spécimens longs de $6-9 \mathrm{~mm}$., rapport 
comme $2: 3$ ), concluant qu'avant de considérer définitivement capense comme une espèce séparée de richiardii, il fallait connaître les dimensions exactes des ventouses de la forme méditerranéenne.

J'ai mesuré les ventouses de la forme méditerranéenne parasite d'Acanthias, d'après un exemplaire (long de 10,5 , large de 8,3 ) mis à ma disposition par R. Dieuzeide; la v. o. mesure longit. 1,75 , transv. 1,95 , la v. v. mesure, 2 à 2,1 de diam. Il n'y a donc aucune raison de conserver capense comme espèce indépendante, ainsi que l'avait, justement, pressenti Odhner.

Je rappelle, avec Odhner (1910, p. 14, note) que le corps des Anaporrhutum est aplati, foliacé et celui des Probolitrema épais, charnu, bombé dorsalement, ventralement plat, même légèrement concave. Cette différence d'habitus, très accentuée, permet, à première vue, d'éviter la confusion.

Outre l'exemplaire récolté par Dieuzeide, j'ai examiné un exemplaire de provenance indéterminée (fig. 1), trouvé parmi les duplicata de l'Institut Zoologique de Strasbourg et aimablement mis à ma disposition par M. le professeur P. Marais de Beauchamp.

Cet exemplaire (monté dans le baume de Canada, après coloration au carmin) est long de $13 \mathrm{~mm}$., large de 11,5 , avec une ventouse orale mesurant longitud. $1 \mathrm{~mm}$., transvers. 1,5, une ventouse ventrale de $2 \mathrm{~mm}$. de diam. La cuticule, jusqu'à l'extré-

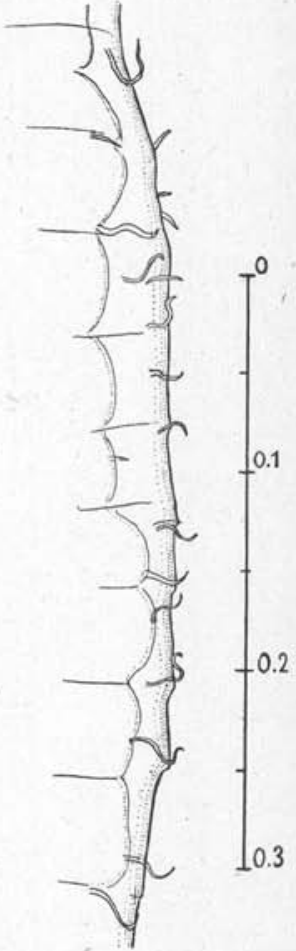

Fig. 2. - Spinules cuticulaires de l'individu de la fig. 1 . mité postérieure, porte des spinules grêles, peu rigides, se rebroussant facilement (fig. 2). On ne voit ni prépharynx, ni œsophage ; le pharynx, longitud. a 0,625 , transvers. 0,875 . Les cæca (1) montrent une indication de légère sacculation, peut-être due en partie à la contraction de l'individu lors de la fixation.

(1) Dans les cæea, il $\mathbf{y}$ avait des œufs en très grand nombre. 
Les testicules sont au nombre de 26-30 de chaque côté et assez serrés, il peut y en avoir deux ou trois contigus au même niveau, mais leur disposition n'est pas régulière. L'ovaire $(0,76 \times 1,02)$ est nettement plus petit que le receptaculum seminis situé en avant de lui. Les vitellogènes sont en tubes contournés, formant, de chaque côté, un peu comme un glomérule.

Les œufs de la fin de l'utérus mesurent $59 \times 52$; mais, plus près de l'ovaire, il y a des œufs plus petits, par exemple mesurant $58 \times 52,56 \times 48,48 \times 44$ et jusqu'à $48 \times 40 \mu(1)$.

\section{Pristiurus melanostomus (Rafinesque 1810)}

A) Georg Mühlschlag (1914, p. 200) a rapporté, avec doute, à Otodistoma veliporum (Creplin) des immatures (métacercaires ?) enkystés dans la paroi de l'estomac de cet hôte (Musée de Berlin).

\section{Famille des CARCHARINIDE}

11. Carcharinus obscurus (Le Sueur 1818) Jordan et Gilbert 1883

La présence signalée par Linton (1901, p. 427, description), de Gasterostoma arcuatum Linton 1900 : 5 grands exemplaires avec œufs et 3 plus petits, dans l'intestin spiral de cet hôte, dans la région de Woods Hole (Massachusetts) semble accidentelle. (Voir Linton 1913, p. 735). L'espèce arcuatum est maintenant considérée comme un Bucephalopsis.

12. Carcharinus milberti (Müller et Henle 1841) Jordan et Gilbert 1883

Syn. Carcharias (Prionodon) Milberti Müller et Henle 1841.

Otodistoma veliporum (Creplin) a été signalé par Diesing (1850, p. 367), sous le nom de Distomum veliporum Crepl, dans l'estomac de cet hôte, à Panorme (Sicile).

\section{Galeus glaucus Rondelet 1554}

Syn. Carcharias glaucus (Linné 1756), G. Cuvier 1817 ; Prionace glauca (Linné 1758), Jordan et Everman 1896.

(1) Pour les æufs, C. Lopez n'a pas indiqué de dimensions, non plus que F. S. Monticelli. Ofenheim (1900, p. 179) a sculement indiqué que le diam. était de $50 \mu$ (et $15-25 \mu$ pour les œufs ovariens).

Looss $(1902$, p. 483) a trouvé, pour les œufs à maturité contenant un miracidium, $64 \times 53 \mu$ et, pour les œufs venant d'être formés, $45 \times 34 \mu$. 
Dans l'estomac de cet hôte, à Gênes, des spécimens de Ptychogonimus megastoma (Rud.) furent trouvés par Willemoes-Suhm (1870, p. $179-181,201$, pl. XI, fig. $4-5$; 1870 , p. $5-7,27$, pl. X? fig. 4-5), qui les étudia sous le nom de Distoma megastoma Rud. Ce fait a été rappelé par C. Parona (1887, p. 490) (cf. Stossich 1888, p. 99).

\section{4 a. Thalassorhinus rondeleti (Risso 1810)}

Syn. Carcharias rondeleti (Risso 1810) Risso 1826.

Le catalogue de la collection Corrado Parona $(1896$, p. 3$)$, a fait mention d'un «Distomum n. sp. ? 》, de Carcharias rondeleti, Italia. Ce distome, qui avait été récolté sur les branchies, à Gênes (22-3-1883), fut décrit sous le nom de Distoma continuum V. Ariola (1899, p. 134-136, 138, pl. V, fig. 6).

\section{4 b. "Carcharias sp. 》}

T. Odhner (1911, p. 516) cite Carcharias sp. parmi les hôtes de la Méditerranée ou de l'Atlantique sud européen lui ayant fourni des Otodistoma veliporum (Creplin).

\section{Eugaleus galeus (Salviani 1554-Linné 1758) Gill 1864}

Syn. Galeus canis Rondelet 1554 ; Squalus galeus Linné 1758 ; Galeus vulgaris Fleming 1828.

Ptychogonimus megastoma (Rud.) a été signalé chez cet hôte par : C. A. Rudolphi $(1819$, p. 102, 387) sous le nom de Distoma. megastomum Rud., dans l'estomac, à Rimini (un des spécimens types a été figuré par Bremser 1824, p. 8, pl. IX, fig. 7-8) (Voir aussi Bremser in Blainville 1824, p. 133); S. Jacoby (1899, p. 18), sous le nom de Distomum megastomum Rud., dans l'estomac, côtes de l'Afrique du Nord.

\section{Famille des GALEORHINIDAE}

16. Galeorhinus mustelus (Linné 1758) Blainville 1816

Syn. Galeus asterias Rondelet 1554; Squalus mustelus Linné 1758 pars; Mustelus plebejus Bonaparte 1841 ; M. vulgaris Müller et Henle 1841 pars.

A) Fr. Sav. Monticelli (1893, p. 139) informe que, chez cet hốe, du golfe de Naples, a été trouvé, une fois, Distoma richiardii Lopez. 
B) Fr. Sav. Monticelli (1890, p. 426, 442, pl. XXII, fig. 17), à Wimereux (Pas-de-Calais), a trouvé un distome immature, long d'env. $1 \mathrm{~mm}$., chez cet hôte. Un peu plus tard, il le nomma Dist. megalocotyle F. S. Monticelli (1893, p. 52-53 note, 227, pl. VIII, fig. 124, portion du tronc excréteur principal), et ajouta quelques renseignements à la première description. D'après la figure publiée en 1890, il s'agit évidemment d'un Hémiuride sans postabdomen ; il est assez vraisemblable que ce distome était accidentel chez le Mustelus.

C) Ptychogonimus megastoma (Rud.) a été trouvé ou signalé chez ce squale, par : W. Nicoll (1914, p. 471, 484, 503), ainsi que H. A. Baylis et E. Idris Jones (1933, p. 631), sous le nom de Ptychogonimus megastomus (Rud.) dans l'estomac, à Plymouth ;

P. J. Van Beneden $(1870$, p. 6-7, 93, pl. IV, fig. $7 a-7 c)$, scus le nom de Distoma megastoma, dans l'œsophage, l'estomac et l'intestin, sur la côte de Belgique ;

Fr. Sav. Monticelli (1890, p. 426), sous le nom de Distomum megastomum Rud., dans l'estomac, à Arcachon (Gironde) ;

R. Ph. Dollfus (non publié), sous le nom de Pt. megastoma (Rud.), dans l'estomac, sur les côtes de Mauritanie (Théodore Monod leg., avril et mai 1923) ;

K. W. Eysenhardt (1829, p. 145-146), sous le nom de Distoma megastomum Rud., dans l'estomac, à Sète (Hérault);

Fr. Zschokke (1887, p. 267), sous le nom de Dist. megastomum Rud., dans l'estomac, à Naples ;

F. Basile (1909) (fide C. Parona 1912, p. 38, 39, 361), sous le nom de $D$. soccus Molin ou D. megastoma Rud., à Messine ;

Raf. Molin (1858, p. 129 ; 1859 , p. $402-403$; 1861, p. 203 ), sous le nom de Distomum soccus Molin, dans l'estomac, à Padoue (type dans la collection C. Parona 1896, p. 3);

Sev. Jacoby (1899, p. 16-24, 29-30, pl. II, fig. 13-16), sous le nom de Distomum megastomum Rud., dans l'estomac, à Adria près Rovigno (Istrie).

Michele Stossich (1890, p. 131), sous le nom de Distomum soccns Molin, dans l'estomac, à Fiume ;

Michele Stossich $(1898$, p. 38, 157), sous le nom de Distoma megastomum .Rud. (= soccus Molin), dans l'estomac, à Trieste ;

Corrado Parona $(1896$, p. 3), sous les noms de «D. (Brachylaimus) megastomum Rud.» et «D. (Brachylaimus) soccus Mol. typ., Mustelus sp. », d'Italie ;

E. A. von Hering (1872, p. 147), sous le nom de D. megastomum Rud., dans l'estomac, dans la collection du Musée de Stuttgart ; 
O. von Linstow (1903, p. 353-354, fig. 3-4), sous le nom de Distoma lymphaticum Linstow, du pharynx, dans la collection A. E. Shipley. C'est T. Odhner (1906, p. 64) qui reconnut l'identité de lymphaticum et megastoma.

\section{Gat.forhinus LAEIS (Rondelet 1554)}

Syn. Mustelus lavis (Rondelet 1554), Risso 1826 ; Squalus mustelus Linné 1758 pars.

A) Ptychogonimus megastoma (Rud,) a été signalé à Naples, chez cet hôte, par :

L. Oerly (1885, p. 218 , p!. X, fig. 10 ), sous le nom de Distoma megastomum Rud., dans l'intestin ;

Fr. Zscbokke (1887, p. 267), sous le nom de Dist. megastomum Rud., dans l'estomac et l'intestin spiral ;

N. Maclaren (1903, p. 261), sous le nom de Distomum megastomum Rud., dans l'estomac.

B) Une forme immature (métacercaire?) de distome trouvée enkystée dans la paroi de l'estomac, à Naples, par Norman Maclaren $(1903$, p. 519-522, fig. 1-5) semble devoir être rapportée à Otodistoma veliporum (Creplin).

18. Galeorhinưs antarcticus (Günther 1870), S. Garman 1913

Syn. Mustelus antarclicus Günther 1870 .

A) Probolitrema clelandi T. Harvey Johnston (1934, p. 29-31, fig. 6) a été décrit d'après quelques spécimens de la cavité du corps de ce squale, à Encounter Bay (South Australia).

Chez cette espèce, les vitellogènes ne sont pas complètement extra-cæcaux, ils empiétent sur la face ventrale des cæca: de. même les testicules ne sont pas complètement extra-cæcaux et empiétent sur la face ventrale des cæca. Plutôt que de créer un genre nouveau, T. H. Johnston $(1934$, p. 31) a préféré modifier légèrement la définition du genre.

B) Probolitrema simile T. Harvey Johnston (1934, p. 30, 31-32, fig. 7) a été décrit d'après quelques spécimens du même hôte, de la même localité, récoltés aussi par le $\mathrm{D}^{\mathrm{r}} \mathrm{J}$.-B. Cleland.

Chez simile, les testicules empiétent moins sur la face ventrale des cæca que chez clelandi, mais il peut s'agir là d'une différence individuelle; l'examen d'une série de spécimens, dit Johnston, montrera peut-être des intermédiaires entre simile et clelandi, 
prouvant qu'il s'agit d'une même espèce. La différence de tailie

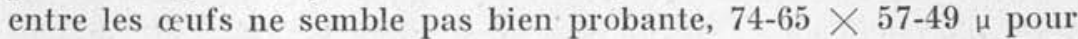
clelandi et $50-42 \times 40-37$ pour simile, à moins qu'il ne soit démontré qu'elle soit liée aux autres caractères invoqués pour la séparation des deux espèces.

C) Probolitrema antarcticum Violet Wcolcock (1935, p. 325-327, pl. IX, fig. 11-14), décrit d'après de nombreux spécimens du coclome de ce Mustelus; un de ces squales contenait 20 spécimens, un second 58 .

Longùeur 5-10,5 (moyenne 7,5), largeur 3-6,5 (moyenne 5). Le diamètre de l'acetabulum est au moins une fois et demie celui de la ventouse orale. Le pharynx a un diamètre transversal de 0,5 , l'œsophage a au moins deux fois la longueur du pharynx. Les cæca intestinaux peuvent présenter quelques légères dilatations ou faibles sacculations augmentant avec la contraction individuelle, mais il n'y a pas réellement de diverticules. En dehors des cæca, il y a, de chaque côté, ventralement, 24-30 testicules de formes irrégulières, lobés ou non ; ils débutent antérieurement à peu près au niveau de la mi-longueur du corps et s'étendent presque jusqu'à l'extrémité postérieure des cæca.

L'ovaire, tri ou pluri lobé, est à peu près médian, immédiatement en arrière de la mi-longueur du corps, il est séparé par le vitelloducte transverse du receptaculum seminis, globuleux, assez gros (diam. 0,75 à $1 \mathrm{~mm}$.) qui est au milieu de la longueur du corps, très légèrement déplacé vers la droite, comme aussi l'ovaire.

Au niveau de la mi-longueur du corps, mais en dehors des cæca, et directement en avant des testicules, se trouvent les vitellogènes formant, de chaque côté, un petit groupe branchu, irrégulièrement digitiforme.

La glande de Mehlis est sur la ligne médiane, du côté gauche de l'espace séparant l'ovaire du receptaculum seminis. V. Wooleock a observé sur le vivant que des œufs pouvaient être refoulés en grand nombre dans le receptaculum seminis. L'ootype se continue, à sa sortie de la glande de Mehlis, par l'utérus, qui décrit quelques sinuosités descendantes jusqu'à l'extrémité postérieure du corps, puis quelques sinuosités ascendantes jusqu'à l'acetabulum et ensuite passe à gauche de l'acetabulum pour. rejoindre (en devenant métraterme) le pore génital q situé immédiatement à côté du pore génital $\delta$, sur la ligne médiane, à mi-distance de la bifurcation œsophagienne et du bord antérieur de l'acetabulum. Le conduit terminal ơ est dilaté en une petite vésicule séminale piriforme, précédant le court canal éjaculateur. Environnant le canal éjaculateur et le métraterme, se trouve un amas de cellules glandulaires pouvant être considérées comme prostatiques.

Les œufs sont nombreux et mesurent $36-45$ sur $35-40 \mu$. 
D'après V. Woolcock (1935, p. 329), P. antarcticum V. W. diffère considérablement de $P$. clelandi T. H. Johnston et $P$. simile T. H. Johnston, en particulier par le rapport des ventouses, la cuticule nue et la forme des testicules ; il diffère moins de $P$. rotundatum T. H. Johnston, mais s'en distingue cependant par son long et étroit œsophage, sa plus grande glande de Mehlis, ses œufs plus petits et plus arrondis, son utérus bien moins développé et ses vitellogènes disposés comme dans le type du genre, alors qu'ils ont une disposition semblant un peu différente chez rotundatum.

Dans quelle mesure s'agit-il de variations individuelles ? C'est ce qu'il n'est pas possible de préciser actuellement, mais il semble peut-être difficile d'admettre qu'il existe trois espèces bien séparées de Probolitrema chez le Galeorhinus (= Mustelus) antarcticus (Günther), des côtes australiennes.

\section{Famille des HETERODONTIDE (CENTRACIONTIDAE)}

\section{Heterodontus philippi (Schneider 1801), Blainville 1816}

Syn. Cestracion philippi (Schneider 1801), Cuvier 1817 ; Centracion philippi (Schneider 1801), S. Garman 1913.

Probolitrema philippi Violet Woolcock (1935, p. 323-325, fig. texte 3, pl. IX, fig. 9-10), dans le cœlome d'un «Port Jackson Shark », de Port Philip Bay (Victoria). Un seul spécimen a été trouvé ; long $6 \mathrm{~mm}$., largeur $3 \mathrm{~mm}$. 5 .

Le diamètre de l'acetabulum est approximativement le double de celui de la ventouse orale. De chaque côté, il y a environ 24 testicules, grands, de formes irrégulières, lobés ou non, serrés, s'étendant ventralement aux caca sur une distance égale à environ la mi-longueur du corps, sans atteindre tout à fait l'extrémité postérieure ; les plus antérieurs dépassent antérieurement l'ovaire et atteignent les vitellogènes.

Les vitellogènes sont constitués, de chaque côté, par un petit groupe de follicules, situé vers la mi-longueur du corps, un peu en dehors des cæca et ventralement. La glande de Mehlis est très petite, médiane, immédiatement en arrière de la moitié de la longueur du corps. L'ovaire, petit, lobé, est situé transversalement, légèrement à droite de la ligne médiane, à un niveau immédiatement postérieur à la glande de Mehlis. Le receptaculum seminis, volumineux, globuleux, est contigu, antérieurement, à l'acetabulum et, postérieurement, à l'ovaire; par son bord postérieur gauche, il touche la glande de Mehlis; il est situé au même niveau que les vitellogènes, à la mi-longueur du corps et immédiatement à droite de la ligne médiane. L'utérus développe ses sinuosités dans l'espace intercæcal, depuis le niveau de la glande de 
Mehlis presque jusqu'à l'extrémité postérieure du corps; la partie terminale de l'utérus passe dorsalement à l'acetabulum, du côté gauche, pour rejoindre directement le pore génital : celui-ci est médian, à mi-distance de la bifurcation œsophagienne et du bord antérieur de l'acetabulum.

Les œufs. mesurent de 48 à $60 \mu$ de long sur 36 à 42 de large ; quelques-uns sont presque sphériques avec un diamètre de 30 à $46 \mu$.

La vésicule séminale, sur la ligne médiane, un peu en avant du bord antérieur de l'acetabulum, est très petite, courte, tubuleuse et légèrement contournée; le canal éjaculateur n'est que faiblement développé.

S'agit-il réellement d'une espèce indépendante? N'est-ce pas plutôt un spécimen, un peu plus allongé que le type, le $P$. rotundatum T. H. Johnston?

La cuticule est nue comme chez $P$. antarcticus V. Woolcock, alors qu'elle est finement épineuse-squameuse chez $P$. clelandi T. H. Johnst. et P. simile T. H. Johnst.

\section{Famille des SQUALIDE}

Squalus acanthias (Rondelet 1554), Linné 1754

\section{Syn. Acanthias vulgaris Risso 1826.}

A) Dans la cavité viscérale de ce squale, à Pise (Italie), Richiardi trouva quelques spécimens d'un distome qui fut décrit sous le nom de Distoma richiardii Corrado Lopez (1888, p. 137-138). Pen après, Fr. Sav. Monticelli (1889, p. 132-134) publia quelques renseignements nouveaux sur l'anatomie de ce distome, d'après de nombreux individus récoltés par Lo Bianco dans la cavité du corps d'Acanthias, du golfe de Naples, et un individu qu'il avait lui-même trouvé à Naples, en 1886, dans la cavité du corps du même hôte. Ce distome n'est pas rare à Naples chez Acanthias et S. Lo Bianco $(1899$, p. $477 ; 1909$, p. 567) l'y a souvent récolté.

E. Ninni $(1900$, p. 57) a cité ce distome, trouvé dans la cavité abdominale d'A. vulg., parmi les récoltes de A. P. Ninni, à Venise.

J'ai fait mention antérieurement (voir R. Ph. D., 1935, p. 200) d'un spécimen trouvé à Alger, par le $D^{r} R$. Dieuzeide, chez le même hôte ; j'ai indiqué plus haut (p. $63, \S 9 \mathrm{~b}, \mathrm{~B}$ ) les caractéristiques de ce spécimen.

Fr. Sav. Monticelli, dans de nombreuses publications, eut l'occasion de mentionner, rappeller, discuter divers caractères structuraux de Dist. richiardii Lopez (voir en particulier : Monticelli, 
1892 , p. 5-8, noyau vitellin de l'œuf ; 1893 passim et p. 139-148, $216,223,225,226,227-228$, pl. I, fig. 10 , pl. V, fig. $51-55$, pl. VI, fig. 87 , pl. VII, fig. 91-102, pl. VIII, fig. 125-130 (1).

Assez souvent, Dist. richiardii Lopez a été utilisé pour diverses recherches histologiques et cytologiques (2).

Les affinités naturelles et la position systématique de D. richiardii Lopez retinrent longtemps l'attention de Looss ; tout d'abord Looss $(1901$, p. 204, 206, 209 ; 1901, p. 557, 558) accepta pour le distome de Lopez le genre Anaporrhutum où l'avait placé Ofenheim.

Lcoss $(1901$, p. 557, 558) dit alors avoir pu étudier des spécimens conservés, mais il n'en indiqua pas la provenance. L'année suivante, l'examen de toutes les caractéristiques structurales d'Anaporrhutum richiardii (Lopez) fut repris par Looss (1902, p. 479, $482,483,484,621,622,791,844,848,852,854,855)$ comparativement à celles d'A. albidum Ofen. et de divers autres distomes. Looss $(1902$, p. 853$)$ indiqua alors que les spécimens d'A. richiardii (Lopez) qu'il avait étudiés, lui avaient été envoyés de Naples par Monticelli et de Gênes par C. Parona (3). Comme conclusion de cet examen comparé, Looss (1902, p. 855) rejeta A. richiardii (Lopez) du genre Anaporrhutum et le choisit pour type du nouveau genre Probolitrema Looss 1902, p. 855.

B) D'après C. Parona (1912, p. 295), Ptychogonimus megastoma (Rud.) aurait été trouvé à Pise, chez Acanthias, par Alceste Arcangeli (1907) ; je n'ai pu consulter l'ouvrage d'Arcangeli, mais je suppose qu'il s'agit d'un lapsus, car Parona (1912, p. 506) ne cite pas Acanthias dans la liste des hôtes de ce distome.

C) Chez Acanthias, Otodistoma veliporum (Creplin) a été signalé sous les noms de :

Distoma microcephalum Baird, par Baird (1855, p. 58, 124, pl. II, fig. $2-2 a ; 1853 b$, p. 22 ; 1855 , p. 73-74) dans l'estomac, à Fal-

(1) Je ne peux pas, ici, m'étendre outre mesure, sur toutes les publications où il est question, plus ou moins accessoirement, de Dist. richiardii Lopez.

On trouvera, dans le catalogue de Stiles et Hassall (1908, p. 227) l'indication de toutes les pages où est cité ce distome, dans les diverses publications de Monticelli ; de même, l'indication de toutes les pages où Looss, dans son ouvrage de 1894 , a fait allusion à ce distome.

(2) Voir par exemple Cesare Créty (1892, p. 24-26 : système nerveux central et éléments ganglionnaires des ventouses, organes tactiles de la cuticule de la ventouse antérieure ; 1892 , p. 373 et 1893 , p. $382-384$ id. $)$; $(1892$, p. $92-97$ : noyau vitellin dans l'œuf ovarien ; 1892 , p. 373 et 1892 , p. 396-399 id.). Le matériel étudié par Créty avait été récolté à Naples, ainsi que le dit Créty, 1892, p. 396.

La structure des ventouses de ce distome fut aussi étudiée par Alceste Areangeli (1907, p. 578-579), fide C. Parona (1911, p. 24).

(3) Le catalogue de la collection C. Parona (1896, p. 3) fait mention de « Distomum (Brachylaimus) Richiardii Lopez », d'Acanthias vulgaris, Italia. 
mouth (synonymie fide Monticelli 1889 , p. $132 ; 1889$, p. $322 ; 1893$ p. 52, note 2).

Distoma veliporum Creplin, par Monticelli (1889, p. 321) dans l'estomac.

O. veliporum (Creplin), par E. M. Layman (1930, p. 76), dans l'estomac, à Vladivostock.

21. Scymnodon ringens Bocage et Capello 1864

Syn. Centroscymnus obscurus Vaillant 1888.

A) P. J. Van Beneden (1870, p. 17, note 1) a annoncé que son fils avait trouvé chez ce squale, des côtes du Portugal, « un grand Distome qui habitait les fosses nasales (Distoma ligula, sp. n.) ». Ce distome a été cité par Rud. Leuckart (1871, p. 450) sous le nom de «Distoma ringens Van Beneden », de même par Linstow (1878, p. 282 « rigens », 360 " ringens »), par V. Ariola (1899, p. 136, « rigens »), par Monticelli (1893, p. 53, « rigens »), etc...

Famille des SCYMNORHINIDE

\section{Scymorhinus hichia (Bonnaterre 1788)}

Syn. Scymnus nicæensis (Risso 1810); S. lichia (Bonnaterre 1788).

Otodistoma veliporum (Creplin) a été signalé chez cet hôte, sous le nom de Dist. veliporum Crepl. par : Fr. Zschokke, 1887, p. 266), dans l'estomac, à Naples ;

Fr. Sav. Monticelli (1889, p. 321), à Madère ;

Fr. Sav. Monticelli (1893, p. 191), dans l'estomac, à Naples,

et sous le nom d'Otodistoma veliporum (Creplin), par G. Mühlschlag (1914, p. 199) (Musée de Berlin).

J'ai personnellement identifié à veliporum (Creplin) des spécimens d'un Otodistoma trouvés dans l'estomac de cet hôte, à Biarritz (Basses-Pyrénées) par Paul Arné (16-5-1935); ces spécimens étaient intermédiaires à veliporum veliporum et veliporum pachytheca, mais très nettement plus proches de pachytheca.

23. Somniosus microcephalus (Schneider 1801)

Syn. Lamargus borealis (Scoresby 1820).

Otodistoma veliporum (Creplin) a été signalé chez cet hôte par G. Mühlschlag (1914, p. 199) (Musée de Berlin). 
Famille des ECHINORHINIDAE

24. Echinorhinus BRucus (Bonnaterre 1788)

Syn. Echinorhinus spinosus (Gmelin 1789); Scymnus spinosus (Gmelin 1789), G. Cuvier 1817.

Otodistoma veliporum (Creplin) a été signalé chez cet hôte sous les noms de: à Nice ;

- Dist. scymna Risso, par Risso (1826, p. 262), dans l'estomac,

- Dist. scymna Risso = insigne Dies., par Villot (1875, p. 477 ; 1878 , p. $3-18$, $39-40$, pl. V, fig. 8 , pl. VI. fig. 1-9), dans l'estomac, à Roscoff.

Dist. veliporum Creplin, par M. Stossich (1890, p. 51) et E. Ninni (1900, p. 57), dans l'intestin, à Venise ; par Fr. Sav. Monticelli $(1889$, p. 133 , note ; 1893, p. 191), dans l'estomac, à Naples ; par Fr. Sav. Monticelli (1893 d, p. 173), au Musée de Palerme.

-- Dist. insigne Dies., par Emilio Ninni (1900, p. 57), dans la collection Ninni.

Otodistoma veliporum (Creplin), par T. Odhner (1911, p. 516), de la Méditerranée ou de l'Atlantique sud-européen; G. Mühlschlag (1914, p. 199), au Musée de Berlin. 